\title{
FACTORES ASOCIADOS A LA NO ADHERENCIA DEL TRATAMIENTO ANTIRRETROVIRAL EN PACIENTES CON VIH EN UN hOSPITAL DE PARAguaY
}

\author{
FACTORS ASSOCIATED WITH NON-ADHERENCE TO ANTIRETROVIRAL \\ TREATMENT IN A HOSPITAL OF PARAGUAY \\ César A. Urizar', Magali Jarolin-Montiel', Nicolás Ayala-Servin', Claudia Centurión-Wenninger',
} Dora Montiel-Garcete ${ }^{2}$.

' Facultad de Ciencias Médicas, Universidad Nacional de Asunción. San Lorenzo, Paraguay.

${ }^{2}$ Hospital Nacional de Itauguá. Itauguá, Paraguay

Correspondencia a:

Nombre: César A. Urizar

Correo electrónico: cesarurizar1994@gmail.com

Telf. y celular: +595972652052

ORCID: https://orcid.org/0000-

0003-2386-2749

https://orcid.org/0000-00017839-858X

https://orcid.org/0000-00032783-2934

https://orcid.org/0000-00029974-8670

https://orcid.org/0000-0003 1587-7836

Palabras clave: : Cumplimiento de la medicación; Negativa del paciente al tratamiento; VIH

Keywords: Medication Adherence; Treatment Refusal; HIV.

Procedencia y arbitraje: no comisionado, sometido a arbitraje externo

Recibido para publicación: 28 de abril 2020

Aceptado para publicación: 27 de diciembre 2020

Citar como:

Urizar CA, Jarolin-Montiel M, Ayala-Servin N, CenturiónWenninger C, Montiel-Garcete D. Factores asociados a la no adherencia del tratamiento antirretroviral en pacientes con vih en un hospital de paraguay. Rev Cient Cienc Med 2020; 23(2): 166-174

\section{RESUMEN}

Introducción: La terapia antirretroviral de gran actividad (TARGA) es la base del tratamiento de pacientes con infección por el virus de la inmunodeficiencia humana $(\mathrm{VIH})$ y, hoy en día, la principal preocupación en relación con el control del $\mathrm{VIH}$ constituye la adherencia efectiva al tratamiento, para un tratamiento efectivo y una apropiada evaluación de los regímenes terapéuticos para poder realizar medidas correctivas y estratégicas. Objetivo: Identificar los factores asociados a la falta de adherencia al tratamiento antirretroviral en pacientes infectados con VIH que acuden al Hospital Nacional de Itauguá, septiembre 2016.

Métodos: Estudio observacional, analítico, corte transversal, muestreo no probabilístico de casos consecutivos. Se incluyeron 42 pacientes infectados con VIH con tratamiento antirretroviral en el Hospital Nacional de Itauguá. Se utilizó encuesta demográfica y cuestionario SMAQ para evaluar la adherencia al tratamiento, para el análisis se realizó la prueba Odds Ratio (OR) y se consideró una $\mathrm{p}<0,05$ como significativa.

Resultados: Un total de 36 pacientes (86\%) no son adherentes a su tratamiento. Se encontró una asociación significativa con presencia de efectos adversos (OR: 8,29, IC95\%: I, 25-54,7I, $p=0,044$ ) y el olvido de la toma de la medicación durante el fin de semana (OR: 28,82, IC95\%: I,45-556, I, p=0,003).

Conclusión: La tasa de falta de adherencia al tratamiento antirretroviral de los pacientes fue alta, y los factores asociados fueron la presencia de efectos adversos y el olvido de la toma de la medicación los fines de semana.

\section{ABSTRACT}

Background: Highly active antiretroviral therapy (HAART) is the basis of treatment for patients with human immunodeficiency virus (HIV) infection and, today, the main concern regarding HIV control is effective adherence to treatment, for effective treatment and appropriate evaluation of therapeutic regimens in order to carry out corrective and strategic measures.

Aim: Identify the associated factors to the lack of adherence to antiretroviral treatment in HIV patients that attend to the National Hospital of Itauguá on September 2016

Methods: Observational and analytical study, transverse cut, non-probabilistic sampling of consecutive cases. The study included 42 HIV patients under antiretroviral treatment of the Itaugua National Hospital. It used a demographic questionnaire and the SMAQ test to evaluate the treatment adherence. For data analysis, it was used Odd Ratio and $p<0,05$ was considered as significant.

Results: 36 patients (86\%) showed non-adherence to their treatment. It was found a significant association with adverse effects presence (OR: 8.29, IC95\%: I.25-54.7I, $\mathrm{p}=0.044)$ and forgetting to take their medicine on weekends (OR: 28.82, IC95\%: I.45-556.I, $\mathrm{p}=0.003$ ).

Conclusion: The rate of non-adherence to antiretroviral treatment in patients was high, and the associated factors were the presence of adverse effects and forgetting to take the medication on weekends.

\section{INTRODUCCIÓN}

a terapia antirretroviral de gran actividad -(TARGA) es la base del tratamiento de pacientes con infección por el virus de la inmunodeficiencia humana (VIH), y consiste en la supresión de la replicación del virus utilizando una combinación de tres o más fármacos antirretrovirales. Este es un componente esencial en la prolongación de la vida y en la mejoría de la calidad de vida de los pacientes infectados con VIH. La supresión adecuada requiere apego estricto a los regímenes prescritos de fármacos antirretrovirales ${ }^{1}$. Con el 
advenimiento de las terapias antirretrovirales se ha demostrado aumentar la sobrevida de los pacientes, aumentando el conteo de CD4 y disminuir la carga viral hasta ser indetectable, y ha disminuido la tasa de mortalidad por VIH, que en décadas pasadas constituyó una verdadera epidemia².

Hoy en día, la principal preocupación en relación con el control del $\mathrm{VIH}$ constituye la adherencia efectiva al tratamiento, donde el paciente toma su medicación siguiendo un horario prescrito y recibe un seguimiento por parte del médico. Esto supone el fracaso o el éxito de la terapéutica 3,4.

EI TARGA facilita la adherencia al tratamiento al informar al paciente sobre los efectos adversos de la medicación y las interacciones farmacológicas de riesgo ${ }^{4}$. La"no adherencia" al tratamiento contribuiría al desarrollo de cepas resistentes a las drogas y a la transmisión del virus durante comportamientos de riesgo ${ }^{5}$.

La correcta adherencia al tratamiento antirretroviral en pacientes infectados con $\mathrm{VIH}$ es importante para un tratamiento efectivo y una apropiada evaluación de los regímenes terapéuticos para poder realizar medidas correctivas y estratégicas (ej.: prescripción ante efectos adversos del medicamento actual; si existe rechazo del diagnóstico por parte del paciente, realizar un trabajo interdisciplinario con un psicólogo; etc.).

La evaluación de la adherencia debe ser parte de la asistencia en el seguimiento del paciente. Se hace estrictamente necesario para el médico identificary tratar, las situaciones concomitantes que pueden impedir una adherencia correcta.

En Paraguay no se encontraron trabajos de investigación relacionados con este tema. En un estudio realizado en Colombia en 2016 se encontró que solo $57,1 \%$ de los encuestados tenían adherencia al tratamiento6. Con el presente trabajo de investigación nos planteamos la interrogante de cuáles son los factores asociados a la falta de adherencia al tratamiento antirretroviral en pacientes infectados con VIH que acuden al Hospital Nacional de Itauguá.

\section{MATERIALES Y MÉTODOS}

Se realizó un estudio cuantitativo, observacional, analítico de corte transversal. El mismo fue llevado a cabo en el Hospital Nacional de Itauguá, localizado en el departamento Central de Paraguay, en el año 2016. La población estudiada fueron pacientes con diagnóstico de infección por VIH que acudían al consultorio externo de Clínica Médica para control médico en el Hospital Nacional de Itauguá y que además recibían sus medicamentos para el tratamiento antirretroviral, desde la cuarta semana de agosto a la quinta semana de septiembre de 2016.

Fueron incluidos pacientes mayores de 18 años que acudían a su control médico periódico y que retiraban sus medicamentos exclusivamente del dispensario del Hospital Nacional de Itauguá. Resultaron excluidos aquellos pacientes que recibían su tratamiento en otros centros asistenciales, además de los que habían dejado el cuestionario incompleto o en blanco.

Se realizó un muestreo no probabilístico de casos consecutivos. Para calcular el mínimo de pacientes incluidos en el estudio se tomó como referencia un estudio de prevalencia en Perú donde se evaluaron los efectos adversos y la adherencia al tratamiento de los pacientes ${ }^{7}$, utilizando la fórmula de proporciones poblacionales para estudios descriptivos, resultando como mínimo una muestra de 42 pacientes ( $z a=1,96, p=0,489, a=0,05, w=0,30)$.

Se realizó entrevistas individuales con los pacientes que acudieron a su control médico mensual en el Servicio de Clínica Médica en el tiempo que abarcó el estudio. Una vez finalizado el control médico, se invitó a los pacientes a participar del estudio, con previa firma de consentimiento informado, realizando las preguntas dirigidas para completar el cuestionario del grupo investigador y el cuestionario SMAQ.

Se utilizó el cuestionario de adherencia SMAQ (Simplified medication adherence questionnaire), desarrollado por el Grupo Español para el Estudio Multifactorial de la Adherencia (GEEMA). El cuestionario SMAQ es un instrumento breve y sencillo, basado en preguntas al propio paciente sobre su hábito en la toma de medicación, validado para la medida de adherencia en pacientes en tratamiento con antirretrovirales8. Se consideró como no adherentes al tratamiento a los pacientes de acuerdo con el resultado del cuestionario. El cuestionario SMAQ contiene 6 preguntas con respuestas cerradas; de acuerdo con las 
respuestas se valora el grado de adherencia del paciente. El cuestionario está validado, este presenta $72 \%$ de sensibilidad y un $91 \%$ de especificidad ${ }^{8,9}$.

Con este cuestionario SMAQ, es considerado "positivo" a un paciente o"no adherente" cuando hay una respuesta afirmativa a cualquiera de las preguntas de este, más de dos dosis no tomadas en la última semana o más de 2 días sin medicación en los últimos 3 meses.

Se tomaron como variables dependientes a los componentes del cuestionario SMAQ8, las cuales son: adherencia al tratamiento (sí, no), olvido de la toma del medicamento alguna vez (sí, no), toma del medicamento a la hora indicada (sí, no), cese de la medicación ante efectos adversos (sí, no), olvido de toma del medicamento en el fin de semana sí, no), número de dosis no tomadas en la última semana (número de pastillas por semana), número de días completos sin tomar la medicación (días).

Las variables independientes fueron: edad (años), sexo (masculino, femenino), estado civil (soltero, casado, divorciado, viudo), educación (ninguna, primaria, secundaria, superior), ocupación laboral (sí, no), procedencia (Central, Cordillera, Paraguarí, Alto Paraná, Itapúa, Amambay, Guairá), domicilio fijo (sí, no), uso de drogas ilícitas en los últimos 6 meses (sí, no), tipo de droga utilizada en los últimos 6 meses (marihuana, cocaína, heroína, etc.), orientación sexual (heterosexual, homosexual, bisexual), efectos secundarios en el último mes al momento de la consulta (sí, no), motivos de cumplimentación incorrectas: (Olvido, Dificultad horaria, Creo que los medicamentos tienen demasiados efectos negativos, No tengo información suficiente sobre ellos, Conozco

Tabla 1. Caracterización sociodemográfica de los pacientes con virus de inmunodeficiencia humana del Hospital Nacional de Itauguá, 2015-2016 (n=42)

$\begin{array}{cccc} & \text { Variable } & \mathbf{n} & \text { Porcentaje (\%) } \\ \text { Sexo } & & & 47,61 \\ & \text { Masculino } & 20 & 52,38 \\ & \text { Femenino } & 22 & \end{array}$

\begin{tabular}{|ccc|}
\hline Estado civil & & 52,38 \\
Soltero & 22 & 26,19 \\
Casado & 11 & 2,38 \\
Divorciado & 1 & 2,38 \\
Viudo & 1 & 16,67 \\
Unión libre & 7 & \\
& & \\
Ocupación laboral & & 66,67 \\
Sí & 28 & 33,33 \\
No & 14 & \\
& & 57,14 \\
Procedencia & & 26,19 \\
Central & 24 & 7,14 \\
Cordillera & 11 & 2,38 \\
Paraguarí & 3 & 2,38 \\
Alto Paraná & 1 & 2,38 \\
Itapúa & 1 & 2,38 \\
Amambay & 1 & \\
Guairá & 1 & 92,85 \\
Sijo & & 7,15 \\
Sí & 39 & \\
No & 3 &
\end{tabular}


a gente que no le ha ido bien, No me gusta tomar medicinas, No he soportado los efectos secundarios del medicamento, voluntad propia).

Para la tabulación de los datos se utilizó el programa Microsoft Excel versión 2010 y para los análisis y pruebas estadísticas, los programas libres Epi Info versión 7 y GNU PSPPIRE versión 0.12 . Se tomó un intervalo de confianza de $95 \%$ y el valor $\mathrm{p}<0,05$ como significativo.

Se utilizaron estadísticas descriptivas como tablas de frecuencias, porcentajes, media y desvío estándar. Para los análisis estadísticos entre variables dicotómicas, se utilizó la prueba no paramétrica de chi cuadrado para la comparación entre grupos, se calculó el Odds Ratio y el intervalo de confianza para ver la magnitud de la asociación. Para la comparación entre una variable cuantitativa y una dicotómica se utilizó la prueba no paramétrica de $U$ de
Mann-Whitney ${ }^{10,11 .}$

Los pacientes participaron de forma totalmente voluntaria y con previa firma de consentimiento informado. La investigación contó con la aprobación del protocolo por la Cátedra de Fisiopatología de la Facultad de Ciencias Médicas de la Universidad Nacional de Asunción para su ejecución.

\section{RESULTADOS}

Un total de cuarenta y dos pacientes fueron encuestados durante el estudio. Entre estos, en su mayoría fueron mujeres, una media de edad de 39 $\pm 12,41$ años (rango: 21-61), y el 45\% entre 21 y 35 años. La mayor parte eran solteros, y en gran porcentaje residentes de Central y Cordillera (Tabla 1).

El $57 \%$ de los pacientes manifestaron haber llegado a terminar sus estudios en primaria

Tabla 2. Respuestas al cuestionario SMAQ de los pacientes con virus de inmunodeficiencia humana del Hospital Nacional de Itauguá, 2015-2016 ( $n=42)$

\begin{tabular}{lcc} 
Variable & n & Porcentaje (\%) \\
$\begin{array}{l}\text { Olvido de la toma del medica- } \\
\text { mento alguna vez } \\
\text { Sí } \\
\text { No }\end{array}$ & 19 & 45,23 \\
\hline $\begin{array}{l}\text { Toma del medicamento a la } \\
\text { hora indicada }\end{array}$ & 23 & 54,77 \\
$\begin{array}{l}\text { Sí } \\
\text { No }\end{array}$ & 24 & \\
\hline $\begin{array}{l}\text { Cese de la medicación ante } \\
\text { efectos adversos } \\
\text { Sí }\end{array}$ & 18 & 57,14 \\
\hline
\end{tabular}

No

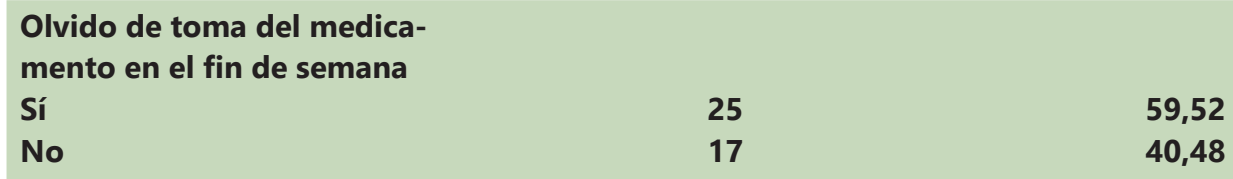

Número de dosis no tomadas Media: 1,21 $\quad$ M1,22
en la última semana

Número de días completos sin tomar la medicación
Media: 0,31 $\pm 0,64$ 
y el $29 \%$ alcanzaron a terminar su educación secundaria, $12 \%$ tenían educación superior y $2 \%$ sin ninguna escolaridad. El $69 \%$ de la población era de orientación heterosexual y el $31 \%$ eran homosexuales.

De entre todos los pacientes, una persona refirió consumo de drogas ilícitas (marihuana). Con el tratamiento antirretroviral recibido el 74\% de los pacientes refirió que sí tuvieron algún efecto adverso en último mes al momento de la consulta . Entre estos efectos, el 54\% refirió trastornos gastrointestinales, 36\% mareos, $17 \%$ insomnio y $12 \%$ mareos.

Se practicó el cuestionario SMAQ a los pacientes para evaluar su adherencia al tratamiento antirretroviral (Tabla 2). Los resultados del cuestionario arrojaron que el $86 \%$ no son adherentes a su tratamiento.

Posteriormente se les preguntó cuáles fueron los motivos de cumplimiento incorrecto del tratamiento antirretroviral (Fig. 1), el olvido y los efectos adversos fueron los principales.

Se hicieron tablas cruzadas de los distintos factores con la falta de adherencia al tratamiento antirretroviral (Tabla 3). Se encontró una asociación significativa con presencia de efectos adversos y el olvido de la toma de la medicación durante el fin de semana.

También se compararon las medianas entre el grupo de los no adherentes y los adherentes con la edad de los pacientes, el número de dosis no tomadas en la última semana y el número de días completos sin tomar su medicación. No se encontraron diferencias significativas en la edad ( $U=98, Z=-0,360, p=0,719)$ y el número de días completos sin tomar la medicación $(U=98$, $\mathrm{Z}=-1,453, \mathrm{p}=0,719)$ con respecto al grupo de los no adherentes, pero sí hubo una diferencia significativa en el número de dosis no tomadas $(U=30, Z=-2,923, p=0,003)$ con respecto a los no adherentes.

\section{DISCUSIÓN}

En el estudio se encontró que entre los factores asociados a la no adherencia estaba el olvido de la toma del medicamento y los efectos adversos del fármaco. La adherencia al tratamiento implica diversos comportamientos por parte del paciente que le permiten hacer frente a un régimen que exige un alto nivel de cumplimiento. En el caso del $\mathrm{VIH}$, tales comportamientos implican tomar

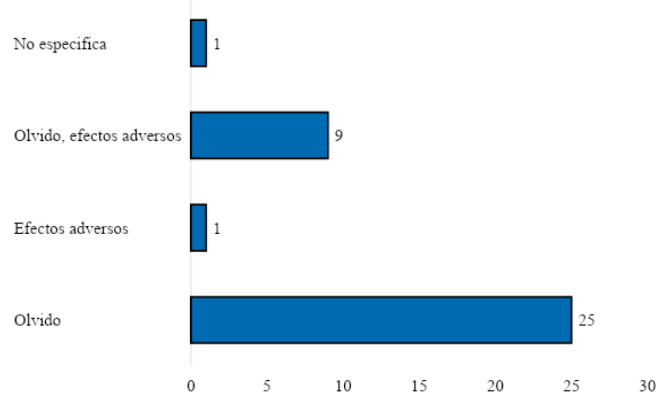

Figura 1: Motivos de cumplimentación incorrecta en los pacientes con virus de inmunodeficiencia humana del Hospital Nacional de Itauguá, 2015-2016 (n=36)

los medicamentos antirretrovirales según las prescripciones médicas (en los horarios indicados, la cantidad exacta y de acuerdo con los intervalos o frecuencias de las dosis); asistir a los controles médicos periódicos y con otros profesionales de la salud; realizarse los exámenes de control, entre otros ${ }^{12}$.

En lo que respecta a la caracterización sociodemográfica los datos obtenidos coinciden con los proporcionados por un estudio realizado por el PRONASIDA (Programa Nacional de Control del SIDA), el cual refiere que la mayor parte de la población con VIH proviene del departamento Central y que prevalece el sexo masculino13. En cuanto al grado de instrucción, la mayoría de los pacientes tenían solo concluidos los estudios de primaria, pero no se encontró una relación entre la falta de adherencia y el nivel de instrucción. De esta manera, en forma general, se ha hallado que los factores sociodemográficos no juegan un rol importante en la no adherencia al tratamiento, así como tampoco la orientación sexual ${ }^{12}$.

La presencia de efectos adversos en los pacientes resultó importante, en estudios de monitoreo de reacciones adversas notificadas se han descrito que un gran número de los pacientes presentaron al menos un efecto adverso, así también dentro de los efectos adversos más prevalentes se destacaron los efectos gastrointestinales ${ }^{14}$. En un estudio realizado en Cali, los pacientes incluidos manifestaron algún efecto adverso durante el tratamiento, se pudoverque los efectos adversos más comunes ante la medicación fueron mareo, rash cutáneo y somnolencia ${ }^{15}$. Debido a que los fármacos se utilizan en combinaciones, el médico debe hacer estrategias terapéuticas adecuadas para lograr una buena adherencia 
al tratamiento antirretroviral y así evitar o minimizar los efectos adversos e interacciones medicamentosas que supondrían un riesgo para la vida del paciente, así lo concluye un estudio hecho en Namibia 4 .

Para evaluar la adherencia se utilizó el cuestionario de adherencia SMAQ, que separa a los adherentes al tratamiento y a los no adherentes $^{6}$. Con este método se encontró que el $86 \%$ de los pacientes no son adherentes al tratamiento antirretroviral. En estudios realizados en Lima, se encontró que la no adherencia al tratamiento antirretroviral fue del $35,9 \%{ }^{6} y$ en otro estudio fue de $48,1 \%{ }^{7}$ que contrastan enormemente con lo hallado en nuestro estudio, esto resulta especialmente preocupante debido a que actualmente los antirretrovirales resultan ser un tratamiento bastante eficaz para el VIH si se lo toma de la manera correcta, ya que requiere una colaboración importante por parte del paciente, quizás la falta de concientización sobre su enfermedad podría ser otro factor importante a la hora de la falta de adherencia al tratamiento.

En un estudio de cohorte realizado en Barcelona16, se vio que realizando una

Tabla 3. Factores asociados con la falta de adherencia al tratamiento antirretroviral en pacientes los pacientes con virus de inmunodeficiencia humana del Hospital Nacional de Itauguá, 2015-2016 (n=42)

\begin{tabular}{|c|c|c|c|c|c|}
\hline Variable & Adherente No & Sí & $\mathrm{OR}^{*}$ & IC $95 \%+$ & $\mathbf{p}$ \\
\hline $\begin{array}{l}\text { Sexo } \\
\text { Masculino } \\
\text { Femenino }\end{array}$ & $\begin{array}{l}18 \\
18\end{array}$ & $\begin{array}{l}2 \\
4\end{array}$ & 2,00 & $0,32-12,33$ & 0.666 \\
\hline $\begin{array}{l}\text { Estado civil } \\
\text { No unidos } \\
\text { Unidos }\end{array}$ & $\begin{array}{c}18 \\
4\end{array}$ & $\begin{array}{c}18 \\
2\end{array}$ & 0,50 & $0,08-3,08$ & 0.380 \\
\hline $\begin{array}{l}\text { Procedencia } \\
\text { Interior } \\
\text { Central }\end{array}$ & $\begin{array}{l}16 \\
20\end{array}$ & $\begin{array}{l}2 \\
4\end{array}$ & 1,60 & $0,26-9,88$ & 0,685 \\
\hline $\begin{array}{l}\text { Grado de instrucción } \\
\text { Ninguna/Escolar } \\
\text { Superior }\end{array}$ & $\begin{array}{c}31 \\
5\end{array}$ & $\begin{array}{l}6 \\
0\end{array}$ & 0,44 & $0,02-8,98$ & 1.000 \\
\hline $\begin{array}{l}\text { Ocupación laboral } \\
\text { No } \\
\text { Sí }\end{array}$ & $\begin{array}{l}11 \\
25\end{array}$ & $\begin{array}{l}3 \\
3\end{array}$ & 0,44 & $0,07-2,53$ & 0.382 \\
\hline $\begin{array}{l}\text { Domicilio fijo } \\
\text { No } \\
\text { Sí }\end{array}$ & $\begin{array}{c}3 \\
33\end{array}$ & $\begin{array}{l}0 \\
6\end{array}$ & 1,35 & $0,06-29,5$ & 0.622 \\
\hline $\begin{array}{l}\text { Orientación sexual } \\
\text { Homosexual } \\
\text { Heterosexual }\end{array}$ & $\begin{array}{l}11 \\
25\end{array}$ & $\begin{array}{l}2 \\
4\end{array}$ & 0,88 & $0,14-5,54$ & 1.000 \\
\hline $\begin{array}{l}\text { Efectos adversos } \\
\text { Sí } \\
\text { No }\end{array}$ & $\begin{array}{c}29 \\
7\end{array}$ & $\begin{array}{l}2 \\
4\end{array}$ & 8,29 & $1,25-54,71$ & 0.044 \\
\hline $\begin{array}{l}\text { Olvido de toma alguna } \\
\text { vez } \\
\text { Sí } \\
\text { No }\end{array}$ & $\begin{array}{l}18 \\
18\end{array}$ & $\begin{array}{l}1 \\
5\end{array}$ & 5,00 & $0,53-47,17$ & 0.198 \\
\hline $\begin{array}{l}\text { Olvido de toma a hora } \\
\text { indicada } \\
\text { Sí } \\
\text { No }\end{array}$ & $\begin{array}{l}19 \\
17\end{array}$ & $\begin{array}{l}5 \\
1\end{array}$ & 0,22 & $0,02-2,11$ & 0.214 \\
\hline
\end{tabular}

Falta de adherencia en pacientes con VIH 
entrevista con el paciente se encuentra que los motivos de la pobre adherencia suelen ser el olvido, la falta de interés y de confianza en el tratamiento; pero con un acompañamiento cercano, en especial del personal de enfermería, la adherencia de la población que era menos del $30 \%$ pasó a ser más del $90 \%$ luego de la intervención. En un estudio realizado en El Salvador se encontró que entre las razones principales de baja adherencia al TARGA en orden de frecuencia están: estar fuera de casa, depresión, el olvido y para evitar los efectos adversos17. Nuestra población refiere que el olvido y los efectos adversos fueron las causas más frecuentes de la falta de adherencia, el acompañamiento más cercano del personal de salud podría tener impacto en la adherencia al tratamiento.

Habría que considerar también los factores de carácter psicológico en estos pacientes porque la ansiedad, la depresión y el estrés dificultan la adherencia adecuada al tratamiento. Se considera necesaria su investigación, el abordaje psicológico y psiquiátrico, sumándose a la terapéutica habitual ${ }^{18 .}$

Con el cuestionario SMAQ también se encontró que un factor muy importante en la falta de adherencia al tratamiento es el olvido de la toma del fármaco durante los fines de semana. En un estudio realizado en Nueva Guinea se constató que la principal razón de dosis no tomadas fue el olvido ${ }^{19}$.

De esta manera, se encontró que existe una relación significativa entre el olvido de la toma del fármaco en los fines de semana y la no adherencia al tratamiento antirretroviral, así como también existe una relación significativa entre la presencia de efectos adversos y la no adherencia al tratamiento, ya que muchos de los pacientes manifestaron cesar el tratamiento ante la presencia de efectos colaterales.

En otros estudios se señalan que el grado de instrucción educativa es también un factor asociado a la falta de adherencia ${ }^{6,7}$, aunque en nuestro trabajo no se encontró una relación significativa, posiblemente debido al pequeño tamaño muestral o puede ser que no sea muy influyente en nuestra población.

Sería de utilidad medir la carga viral y/o el conteo de linfocitos CD4 para hacer el seguimiento adecuado de los pacientes, para tomar las medidas de abordaje adecuadas para mejorar la adherencia al tratamiento, dado que un estudio de cohorte de 6 meses hecho en Brasil encontró que los pacientes que retiraban regularmente su medicación del dispensario tenían una carga viral más baja y mejores niveles de linfocitos CD4 ${ }^{20}$.

Resulta preocupante la alta tasa de no adherencia al tratamiento, sin embargo, entre las limitaciones del estudio se podría mencionar el tamaño de muestra pequeño, por lo que las inferencias serían difíciles extrapolarlas a la población paraguaya. Lo ideal sería realizar un estudio multicéntrico que abarque a todos los hospitales, en coordinación con el PRONASIDA, que pueda reflejar la realidad de la situación a nivel país, y poder llevar un monitoreo cercano sobre la adherencia efectiva de los pacientes paraguayos con $\mathrm{VIH}$ a su tratamiento antirretroviral.

\section{CONCLUSION}

La tasa de falta de adherencia al tratamiento antirretroviral de los pacientes fue alta, y los factores asociados fueron la presencia de efectos adversos y el olvido de la toma de la medicación los fines de semana. Recomendamos realizar mediciones de los niveles de linfocitos CD4 para una valoración de la efectividad del tratamiento y contrastar con los resultados que se obtengan con este cuestionario, además del monitoreo de los efectos adversos y de la salud mental de los pacientes, para así realizar una intervención oportuna y mejorar su calidad de vida. Por último, se necesitan estudios multicéntricos para tener una mejor perspectiva de la realidad actual de la adherencia al tratamiento de este grupo de pacientes. 


\section{REFERENCES}

1.L LD. Harrison: Principios de Medicina Interna. 18.a ed. McGraw Hill Mexico; 2012. $4418 p$.

2.Rodríguez Roque GJ. Respuesta clínica e inmunovirológica a la terapia antirretroviral combinada al año en una cohorte de pacientes con diagnóstico de $\mathrm{VIH}$ atendidos en el hospital Dr. Roberto Calderón Gutiérrez en el periodo Enero-Diciembre 2015 [Internet]. Universidad Nacional Autónoma de Nicaragua; 2017. Disponible en: https://repositorio.unan.edu. ni/4304/1/96901.pdf

3.Abondano Herrera V. Adherencia al tratamiento en pacientes con VIH-SIDA [Internet]. Universidad de La Sabana; 2012. Disponible en: https:/intellectum.unisabana.edu.co/ handle/10818/4541

4.Clapé-Laffita O. Estrategias farmacológicas para facilitar la adherencia a la terapia antirretroviral altamente activa. (Spanish). Pharmacol Strateg to Improv adherence to highly Act Antiretrovir Ther [Internet]. marzo de 2013 [citado 19 de noviembre de 2016];51(2):200-3. Disponible en: https://www. medigraphic.com/pdfs/imss/im-2013/im132p.pdf

5.Hansana V, Sanchaisuriya P, Durham J, Sychareun V, Chaleunvong K, Boonyaleepun $S$, et al. Adherence to Antiretroviral Therapy (ART) among People Living With HIV (PLHIV): a cross-sectional survey to measure in Lao PDR. BMC Public Health [Internet]. 2013 [citado 21 de agosto de 2016];13:617. Disponible en: http:// dx. doi.org/10.1186/1471-2458-13-617

6.Cardona-Duque DV, Medina-Pérez ÓA, Herrera-Castaño SM, Orozco-Gómez PA. Adherence to antiretroviral treatment and associated factors in people living with HIV/ AIDS in Quindío, Colombia. Rev fac med. 2017;65(3):403-10. Disponible en: http:// www.scielo.org.co/scielo.php? script $=$ sci_arttextcipid =S0120-00112017000300403

7.Pacífico J, Gutiérrez C. Information about the medicines and adherence to high activity antiretroviral treatment in patients with HIV/ AIDS in a hospital of Lima, Peru. Rev Peru Med Exp Salud Publica [Internet]. marzo de 2015 [citado 19 de noviembre de 2016];32(1):6672. Disponible en: http://www.scielosp.org/ scielo. php? script =sci_abstract\& $\mathcal{L}$ id $=S 1726$ -

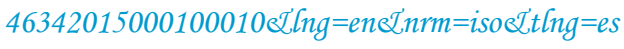

8.Ortega Suárez FJ, Sánchez Plumed J, Pérez Valentín MA, Palomo PP, Muñoz Cepeda MA, Lorenzo Aguiar D. Validación del cuestionario simplificado de adherencia a la medicación (SMAQ) en pacientes con trasplante renal en terapia con tacrolimus. (Spanish) [Internet]. diciembre de 2011 [citado 19 de noviembre de 2016];31(6):690-5. Disponible en: http://scielo. isciii.es/pdf/nefrologia/v31n6/original4.pdf

9.Knobel H, Alonso J, Casado JL, Collazos J, González J, Ruiz I, et al. Validation of a simplified medication adherence questionnaire in a large cohort of HIV-infected patients: the GEEMA Study. AIDS [Internet]. 2002;16(4):605-13. Disponible en: https://journals. Lww.com/aidsonline/ Fulltext/2002/03080/Validation_of_a_simplified_ medication_adherence.12.aspx

10.Hulley SB, Cummings SR, M.D WSB. Diseño de investigaciones clínicas. Wolters Kluwer Health España/Lippincott Williams \& Wilkins; 2014. 417 p.

11.Sampieri $\mathrm{RH}$, Collado CF, Lucio PB. Metodología de la investigación. McGraw-Hill Education; 2014. 600 p.

12.Reyes-Flores E, Trejo-Alvarez R, ArguijoAbrego $S$, Jimenez-Gómez A, Castillo-Castro A, Hernández-Silva $A$, et al. Adherencia Terapéutica: Conceptos, determinantes y nuevas estrategias. Rev Med Hondur [Internet]. 2016;84(3-4):125-32. Disponible en: http:// www.6vs.hn/RMH /pdf/2016/pdf/Vol84-3-4-2016. pdf\#page $=49$

13.Barrios, Cabello, Quiñonez. Informe de la situación epidemiológica del VIH - Paraguay, 2015 [Internet]. Disponible en: http://www. pronasida.gov.py/images/documentos/inf epi vif 2015 final.pdf

14.Morillo Verdugo R, Fernández Lisón LC, Huertas Fernández MJ, Martín Conde MT, Roldan Morales JC, Ruano Camps R, et al. Papel del farmacéutico de hospital en la prevención, identificación y manejo de los efectos adversos asociados al tratamiento antirretroviral. Farm Hosp [Internet]. 2010;34(5):237-50. Disponible en: http://www.sciencedirect.com/science/article/pii/ S1130634310001765

15.Galindo J, Amariles P, Mueses-Marín HF, Hincapié JA, Sebastián González-Avendaño and Ximena Galindo-Orrego. Effectiveness and safety of generic version of abacavir/ 
lamivudine and efavirenz in treatment naïve HIV-infected patients: a nonrandomized, openlabel, phase IV study in Cali-Colombia, 20112012. BMC Infect Dis [Internet]. octubre de 2016 [citado 12 de diciembre de 2016];16:1-13. Disponible en: https://6mcinfectdis.6iomedcentral. com/articles/10.1186/s12879-016-1871-x

16.Navarro J, Pérez $M$, Curran A, Burgos J, Feijoo $M$, Torrella $A$, et al. Impact of an adherence program to antiretroviral treatment on virologic response in a cohort of multitreated and poorly adherent HIV-infected patients in Spain. AIDS Patient Care STDS [Internet]. octubre de 2014 [citado 12 de diciembre de 2016];28(10):53742. Disponible en: https://europepmc.org/article/ med/25111167

17.Betancur MN, Lins L, Oliveira IR de, Brites C. Quality of life, anxiety and depression in patients with HIV/AIDS who present poor adherence to antiretroviral therapy: a crosssectional study in Salvador, Brazil. Brazilian J Infect Dis [Internet]. 2017;21(5):507-14. Disponible en: http://inkinghub.elsevier.com/
retrieve/pii/S1413867016306596

18.Moreno Díaz J, Martínez González A, Crespo Redondo D, Arazo Garcés P. Factores asociados a ansiedad y depresión en pacientes VIH. Rev Multidiscip del SIDA [Internet]. 2017;5(12):34-42. Disponible en: http://www. sidastudi.org/resources/inmagic-img/DD45482.pdf

19.Gare J, Kelly-Hanku A, Ryan CE, David $M$, Kaima $P$, Imara $U$, et al. Factors influencing antiretroviral adherence and virological outcomes in people living with HIV in the Highlands of Papua New Guinea. PLoS One. 2015;10(8):1-15. Disponible en: https:// journals.plos.org/plosone/article? id=10.1371/journal. pone. 0134918

20.Gerenutti M, Vieira Martinez AM, Bergamaschi $C$ de $C$. The Effectiveness of a Pharmaceutical Care Model on Adherence to Antiretroviral Therapy: A SAME-Based Cohort Study in Brazil. Adv Pharm Bull [Internet]. 2017;7(3):469-72. Disponible en: http://dx.doi. org/10.15171/ap6.2017.056 